\title{
A HETEROGENEOUS INTERPOLANT
}

\author{
WALTER TAYLOR
}

In this note we exhibit an interpolant for a certain valid implication $\vDash \varphi \rightarrow \psi$, where $\varphi$ and $\psi$ come from the infinitary language $L_{\omega_{1} \omega_{1}}$. The existence of this interpolant follows from Takeuti's heterogeneous interpolation theorem [5], but unfortunately the proof in [5] is not explicit enough to allow one to find the interpolant explicitly. Takeuti's theorem asserts the existence of an interpolant in the class $\bar{L}_{\omega_{1} \omega_{1}}$ of heterogeneous formulas, which admits the rules of formation of $L_{\omega_{1} \omega_{1}}$ plus the following additional rule: if $\varphi \in \bar{L}_{\omega_{1} \omega_{1}}$ and $\left\langle Q_{\alpha}\right\rangle_{\alpha<\beta}$ is a sequence of quantifiers (i.e. $Q_{\alpha}=\exists$ or $\left.Q_{\alpha}=\forall\right)$ then $Q_{0} x_{i_{0}} \cdots Q_{\alpha} x_{i_{\alpha}} \cdots_{(\alpha<\beta)} \varphi \in \bar{L}_{\omega_{1} \omega_{1}}$. (The semantic interpretation is the obvious one; consult [2, §C] or [4].)

The present interpolation example (whose investigation was suggested by J. Malitz) will be presented as a definability theorem. Namely we give a formula explicitly defining an isomorphism between two isomorphic well-founded extensional relations.

We take unary predicates $A_{1}, A_{2}$ and binary predicates $E_{1}, E_{2}, F$ (written medially). Let $\sigma$ be the conjunction of the following sentences.

$$
\begin{aligned}
& \forall x\left[\left(A_{1} x \vee A_{2} x\right) \wedge \neg\left(A_{1} x \wedge A_{2} x\right)\right] \\
& \forall x \forall y\left[x E_{i} y \rightarrow A_{i} x \wedge A_{i} y\right] \quad(i=1,2) \\
& \forall x \forall y\left[A_{i} x \wedge A_{i} y \rightarrow\left[x \simeq y \leftrightarrow \forall z\left(z E_{i} x \leftrightarrow z E_{i} y\right)\right]\right] \quad(i=1,2) \\
& \forall x_{0} \forall x_{1} \cdots\left[\neg \bigwedge_{j \in \omega} x_{j+1} E_{i} x_{j}\right] \quad(i=1,2) \\
& \forall x \forall y\left[x F y \rightarrow A_{1} x \wedge A_{2} y\right] \\
& \forall x\left[A_{1} x \rightarrow \exists ! y(x F y)\right] \\
& \forall y\left[A_{2} y \rightarrow \exists ! x(x F y)\right] \\
& \forall u \forall v \forall x \forall y\left[x F y \wedge u F v \rightarrow\left(u E_{1} x \leftrightarrow v E_{2} y\right)\right] .
\end{aligned}
$$

One may easily check that if

$$
\left\langle U ; A_{1}, A_{2}, E_{1}, E_{2}, F\right\rangle \vDash \sigma
$$

Received March, 14, 1972. 
and

$$
\left\langle U ; A_{1}, A_{2}, E_{1}, E_{2}, F^{\prime}\right\rangle \vDash \sigma,
$$

then $F=F^{\prime}$. Thus $F$ is implicitly defined by $\sigma$, and hence by Takeuti's theorem together with the usual argument for Beth's theorem, there is a (heterogeneous) formula $\Phi(x, y)$ such that $\sigma \vDash x F y \leftrightarrow \Phi(x, y)$, with $F$ not appearing in $\Phi$. (Such $\Phi$ cannot be in any $L_{x \lambda}$, as follows from the proof of Malitz [3, Theorem 4.2].) The aim of this note is to explicitly exhibit $\Phi$.

Let $C$ be the set of finite sequences of 0 's and 1's (including the empty sequence $\square$ ). For $\square \neq \sigma=a_{0} a_{1} \cdots a_{n-1} a_{n} \in C$, we let $\hat{\sigma}=a_{0} \cdots a_{n-1}$. For all $\sigma \in C$ we take variables $x_{\sigma}$ and $y_{\sigma}$. For all $\sigma \in C$, let $Q_{\sigma}$ stand for $^{1}$

$$
\left(\forall x_{\sigma 0} \in A_{1}\right)\left(\exists y_{\sigma 0} \in A_{2}\right)\left(\forall y_{\sigma 1} \in A_{2}\right)\left(\exists x_{\sigma 1} \in A_{1}\right) .
$$

Now let $\Phi(x, y)$ be

$$
\left(A_{1} x\right) \wedge\left(A_{2} y\right) \wedge Q_{\square} \cdots \underset{\substack{\sigma, \tau \in C \\|\sigma| \leq|\tau|}}{Q_{\sigma}} \cdots Q_{\tau} \cdots \bigwedge_{\substack{\sigma \in C \\ \sigma \neq \square}}\left(x_{\sigma} E_{1} x_{\hat{\sigma}} \leftrightarrow y_{\sigma} E_{2} y_{\hat{\sigma}}\right)
$$

where $|\sigma|$ denotes the length of $\sigma$. We claim that $\sigma \vDash(\Phi(x, y) \leftrightarrow x F y)$. To see this, let $\mathfrak{U}=\left\langle U ; A_{1}, A_{2}, E_{1}, E_{2}, F\right\rangle$ be any model of $\sigma$. We then need to see that for $a, b \in U, \mathfrak{U} \vDash \Phi[a, b]$ if and only if $(a, b) \in F$. Certainly if $(a, b) \in F$, then we may obviously use the isomorphism $F$ to continue to establish the satisfaction of $\Phi$ in $\mathfrak{A}$. Conversely suppose that for some $a$ and $b, a \in A_{1}$ and $b \in A_{2}$,

$$
\mathfrak{A} \vDash \Phi[a, b] \quad \text { but }(a, b) \notin F .
$$

We let $a$ be $E_{1}$-minimal among those $a$ for which such $b$ exists, and let $b$ be $E_{2}$-minimal such that (*) holds for $b$ and this value of $a$. Notice that $\Phi(x, y)$ is logically equivalent to

$$
\begin{aligned}
& \forall x_{0} \exists y_{0}\left[\left(x_{0} E_{1} x \leftrightarrow y_{0} E_{2} y\right) \wedge \Phi\left(x_{0}, y_{0}\right)\right] \wedge \\
& \forall y_{1} \exists x_{1}\left[\left(x_{1} E_{1} x \leftrightarrow y_{1} E_{2} y\right) \wedge \Phi\left(x_{1}, y_{1}\right)\right] .
\end{aligned}
$$

Thus we know that

$$
\mathfrak{A} \vDash\left[\begin{array}{l}
\forall x_{0} \exists y_{0}\left[\left(x_{0} E_{1} a \leftrightarrow y_{0} E_{2} b\right) \wedge \Phi\left(x_{0}, y_{0}\right)\right] \wedge \\
\forall y_{1} \exists x_{1}\left[\left(x_{1} E_{1} a \leftrightarrow y_{1} E_{2} b\right) \wedge \Phi\left(x_{1}, y_{1}\right)\right]
\end{array}\right]
$$

${ }^{1}$ We express the quantifiers $Q_{\sigma}$ with " $\epsilon$ " for cognitive purposes. Clearly it is possible to write $\Phi$ in strict accordance with the formation rule mentioned above. 
By the minimality of $a$ and $b$, we know that therefore

$$
\mathfrak{U} \vDash\left[\begin{array}{l}
\forall x_{0} \exists y_{0}\left[\left(x_{0} E_{1} a \leftrightarrow y_{0} E_{2} b\right) \wedge x_{0} F y_{0}\right] \wedge \\
\forall y_{1} \exists x_{1}\left[\left(x_{1} E_{1} a \leftrightarrow y_{1} E_{2} b\right) \wedge x_{1} F y_{1}\right]
\end{array}\right] .
$$

But since $F$ is an isomorphism and since $E_{1}$ and $E_{2}$ are each extensional, we see that $(a, b) \in F$.

\section{REFERENCES}

[1] Barwise, J., ed., The syntax and semantics of infinitary languages, Lecture notes in mathematics \#72, Springer-Verlag, Berlin, 1968.

[2] Henkin, L., Some remarks on infinitely long formulas, pp. 167-183 in: Infinitistic methods (Proceedings of the symposium on foundations of mathematics, Warsaw, September, 1959), Pergamon, Państwowe Wydawnictwo Naukowe, Warsaw, 1961.

[ 3 ] Malitz, J., Infinitary analogs of theorems from first order model theory, J. Symbolic Logic 36 (1971), 216-228.

[4] Mycielski, J., On the axiom of determinateness, Fund. Math., 53 (1964), 205-224.

[ 5 ] Takeuti, G., A determinate logic, Nagoya Math. J. 38 (1970), 113-138. (Essentially the same article appears as pp. 237-264 in [1].)

Added July 18, 1973 The following articles give further information on Takeuti's (and other) interpolation theorems:

[6] Kueker, D. W., Löwenheim-Skolem and interpolation theorems in infinitary languages, Bull. Amer. Math. Soc. 78 (1972), 211-215.

[ 7 ] Nebres, B. F., Herbrand uniformity theorems for infinitary languages, J. Math. Soc. Japan 24 (1972), 1-19.

[ 8 ] Swett, A. K., Interpolation theorems for languages with game quantifiers, ms., Toronto, 1973.

University of Colorado 